\title{
EI CASO como dispositivo de las prácticas escolares
}

\author{
The CASE as a Device of School Practices
}

\section{O CASO como um dispositivo para práticas escolares}

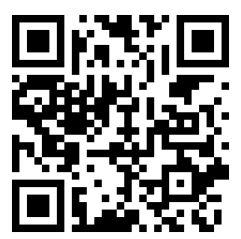

\author{
Lorena Ramírez-Casas del Valle \\ Pontificia Universidad Católica de Valparaíso \\ Viña del Mar, Chile \\ lorena.ramirez@pucv.cl \\ (iD) https://orcid.org/0000-0001-5746-7777 \\ René Valdés-Morales \\ Pontificia Universidad Católica de Valparaíso \\ Viña del Mar, Chile \\ rene.valdes@pucv.cl \\ https://orcid.org/0000-0003-4242-9748
}

Recibido • Received • Recebido: 11 / 01 / 2018

Corregido • Revised • Revisado: 28 / 01 / 2019

Aceptado • Accepted • Aprovado: 06 / 05 / 2019

\begin{abstract}
Resumen: El sistema educativo chileno en los últimos 15 años ha creado nuevos cuerpos normativos y legales para favorecer la inclusión, evitar la discriminación y mejorar la convivencia escolar. Estas leyes interpelan directamente a las escuelas, las cuales desde sus propias traducciones generan también sus propias prácticas educativas. El objetivo de esta investigación fue comprender cómo las escuelas traducen y articulan estas políticas. Mediante una etnografía de dispositivos (Sisto y Zelaya, 2013), se produjeron entrevistas y observaciones en dos escuelas que cuentan con un equipo específico de profesionales para hacer articular estas políticas. Los resultados indican que desde las prácticas de los equipos emerge, como resultado, la existencia de un dispositivo que hemos denominado CASO, que es una expresión que ocupan ambas escuelas y que guarda relación con estudiantes que presentan problemas conductuales o de aprendizajes. El CASO como dispositivo organiza las labores de los equipos, principalmente en torno a la detección y apoyo de estudiantes, levantamiento de evidencias, y resguardos frente a la fiscalización. Las discusiones giran en torno a los alcances de este dispositivo y a los efectos que genera en la comunidad escolar.
\end{abstract}

Palabras claves: Dispositivo; teoría de actor red; prácticas educativas; políticas educativas. 
doi: http://dx.doi.org/10.15359/ree.23-3.12

URL: http://www.una.ac.cr/educare

CORREO: educare@una.cr

\begin{abstract}
In the last 15 years, the Chilean educational system has created new normative and legal bodies to furthering inclusion, avoid discrimination, and improve school coexistence. These laws directly address schools, which, from their own translations, also generate their own educational practices. The objective of this research was to comprehend how schools translate and articulate these policies. Through an ethnography of devices (Sisto y Zelaya, 2013), interviews and observations took place in two schools that have a specific team of professionals to articulate these policies. The results show that, from the practices of the teams, a device called CASE emerges as a result; this device is an expression that both schools need and is related to students who have behavioralor learning problems. The CASE, as a device, organizes the teams work, mainly around the detection and support of students, evidence gathering, and safeguards facing the audit. The discussions revolve around the scope of this device and the effects it generates in the school community.
\end{abstract}

Keywords: Device; actor-network theory; educational practices, educational policies.

Resumo: Nos últimos 15 anos, o sistema educacional chileno criou novos órgãos normativos e legais para promover a inclusão, prevenir a discriminação e melhorar a convivência escolar. Essas leis desafiam diretamente as escolas que, a partir de suas próprias traduções, geram também suas próprias práticas educacionais. $\mathrm{O}$ objetivo desta pesquisa foi entender como as escolas traduzem e articulam essas políticas. Por meio de uma etnografia de dispositivos (Sisto e Zelaya, 2013), foram produzidas entrevistas e observações em duas escolas que possuem uma equipe específica de profissionais para articular essas políticas. Os resultados indicam que a partir das práticas das equipes emerge, como resultado, a existência de um dispositivo que denominamos CASO, que é uma expressão que ocupa as duas escolas e que está relacionada a estudantes com problemas comportamentais ou de aprendizagem. O CASO como dispositivo organiza o trabalho das equipes, principalmente em torno da detecção e apoio aos estudantes, coleta de evidências e salvaguardas frente a fiscalização. As discussões giram em torno das possibilidades desse dispositivo e dos efeitos por ele gerados na comunidade escolar.

Palavras-chave: Dispositivo; teoria do ator de rede; práticas educativas; políticas educativas.

\title{
Introducción
}

En los últimos años, Chile ha llevado adelante una reforma educacional que ha tenido como objetivo el mejoramiento de la calidad de educación. Para esto ha generado distintos cuerpos legales con sus respectivas normativas que se traducen en políticas públicas que, como tales, buscan modificar o generar desplazamientos en el espacio social. En ese sentido, las escuelas son directamente interpeladas por estas normativas, pues deben traducir estas políticas $y$, a la vez, desplegar diferentes prácticas de actuación (Grau, Íñiguez-Rueda y Subirats, 2010).

En términos declarativos, esta reforma tiene como propósito avanzar hacia un sistema más equitativo, cuestión que exige "un nuevo modelo educacional que sea inclusivo, que amplíe la actual noción restringida de calidad y que la potencie integralmente" (Ministerio de Educación, 2016a, p. 5) en las que se asume que los objetivos de la educación van más allá de propiciar aprendizajes del estudiantado al interior de una sala de clases, apuntan también 
hacia una integración social y formación ciudadana, en la cual el Estado trabaje con una óptica de aseguramiento de los derechos ciudadanos a la educación de calidad. Este interés se ha materializado en la formulación de distintas leyes, normativas y estructuras de regulación sectorial, que como se declara en el documento sobre la Ley de Educación General (LGE) (Ley 20.390), posibiliten la existencia de un aprendizaje permanente, que abarque las distintas etapas de la vida de las personas y permita al estudiantado "alcanzar su desarrollo espiritual, ético, moral, afectivo, intelectual, artístico y físico, mediante la transmisión y el cultivo de valores, conocimientos y destrezas (LGE, art. 2)" (Ministerio de Educación, 2016b, p. 13).

Desde esta perspectiva se encuentra la Ley de subvención escolar (SEP), Ley 20.248 (Ministerio de Educación, 2008); la Política Nacional de Educación Especial, promulgada el año 2005 (Ministerio de Educación, 2005); y la Ley de Convivencia Escolar, Ley 20.536 (Ministerio de Educación, 2011b), junto a la Política de Convivencia Escolar año 2015-2018 (Ministerio de Educación, 2015).

Estas distintitas leyes, normativas y procedimientos programáticos convergen en la escuela, donde entran en una red híbrida de políticas, que se crean y recrean "fruto de múltiples procesos de asociación en los que participan una gran cantidad y diversidad de elementos" (Grau et al., 2010, p. 66) que van siendo parte de las prácticas locales de actuación, desde las que se generan particulares formas de traducción y, por ende, de implementación de estas políticas.

Siguiendo los aportes teóricos de la teoría de actor red (ANT) (Latour, 1998), asumimos que, si bien las prácticas de las escuelas se enmarcan dentro de este corpus legal y político, estas son traducidas desde las propias prácticas de la escuela, las que, a su vez, reconstruyen otras. Por tanto, la implementación de una política no es la mera asimilación y replica de las prescripciones de la política central, sino que opera desde una traducción, proceso que se entiende como un espacio intermedio entre la política y la práctica donde se definen las respuestas de los agentes escolares a la política (Verger, Bonal y Zancajo, 2016).

En esta investigación realizamos una etnografía de dispositivos (Sisto y Zelaya, 2013) y el objetivo fue analizar cómo dos escuelas traducen y articulan esta diversidad de políticas en sus prácticas de escolares. Las escuelas seleccionadas se caracterizan por tener un equipo de apoyo al estudiantado, en el que participan docentes y profesionales que responden a distintas normativas y programas escolares, entre los que se encuentran: el programa de integración escolar (PIE), el programa de convivencia escolar, y el de duplas psicosociales (que se enmarcan en la ley SEP).

\section{Modelo de gestión y políticas educativas}

En las últimas décadas, en distintos países, entre ellos Chile, ha predominado un modelo de gestión educativa en el que priman principios propios de la empresa privada, conocidos con la denominación nuevo managment público (NPM por su sigla en inglés). En Chile, desde 
doi: http://dx.doi.org/10.15359/ree.23-3.12

URL: http://www.una.ac.cr/educare

CORREO: educare@una.cr

el año 1980 se comienza a implementar este modelo de mercado en el campo educativo, lo que implicó cambios en la administración de la educación en distintos niveles como: a) se incorporan privados al sistema educativo, con la consecuente coexistencia de establecimientos dependientes de las municipalidades, y otros pertenecientes a privados (establecimientos particulares subvencionados); b) se instaura un modelo de financiamiento público que funciona como subvención a la demanda; $y, c)$ la calidad educativa es medida mediante una prueba estandarizada denominada Sistema de medición de calidad escolar (SIMCE) (Cornejo, 2006).

A principios del nuevo siglo, en el marco de los movimientos estudiantiles, conocidos con en nombre de revolución pingüina (ocurridos los año 2006 y 2011), se abrieron nuevos debates respecto lo que se entiende por calidad de educación y formas de gestionar lo público, que dieron pie a una reforma educativa, cuya misión ha sido avanzar hacia un sistema más equitativo e inclusivo, que permita ampliar la actual noción restringida de calidad y que la potencie integralmente (Ministerio de Educación, 2016a). Con esta reforma, se asume que el Estado debe asegurar los derechos de la ciudadanía a la educación de calidad, que propicie por una parte, los aprendizajes del estudiantado al interior de una sala de clases, así como también, la integración social y formación ciudadana.

En este contexto de reformas, en el año 2011 se promulga la Ley de Aseguramiento de la Calidad de la Educación, Ley N.o 20.529 (Ministerio de Educación, 2011a). Se suman al Ministerio de Educación dos nuevos organismos. Por una parte, se crea la Superintendencia de Educación Escolar, la cual está encargada de fiscalizar el cumplimento de las normativas educacionales y el buen uso de los recursos. Y por otra parte, se crea la Agencia de Calidad de la Educación, que es la encargada de evaluar el desempeño de las escuelas así como también orientar a aquellas que se encuentran por debajo de los estándares definidos. Con estos propósitos, dicho organismo clasifica todos los años a las escuelas con base en los desempeños del alumnado especialmente en prueba SIMCE, bajo las denominaciones de desempeño alto, medio, mediobajo e insuficiente. Dicha clasificación supone, por un lado, el poder contar con mayor apoyo por parte del Ministerio de Educación en el diseño y ejecución de planes de mejoramiento; pero también implica el posible cierre de aquellas escuelas que se han mantenido en la categoría de desempeño insuficiente por un período de cuatro años, instalándose así la amenaza de cierre de escuelas (Assaél, Acuña, Contreras y Corbalán, 2014).

Junto con esto, la política educacional ha promulgado distintas leyes, y normativas -con sus consecuentes programas- que buscan asegurar el derecho a la educación y permanencia de todo el estudiantado; promover la inclusión de todos los niños y las niñas, así como también propiciar el desarrollo de una convivencia escolar inclusiva, en el marco de una equidad de género y enfoque de derechos (Mineduc, 2015).

En el marco del decreto 170 de la ley 20.2011 (Ministerio de Educación, 2007) que elabora nuevos lineamientos para subvencionar las necesidades educativas especiales transitorias, se 
encuentra el Programa de Integración Escolar (PIE), el cual es una estrategia del sistema escolar que tiene el propósito de entregar apoyos adicionales a los estudiantes que presentan necesidades educativas especiales. Por lo tanto, no se dirigen a toda la población que eventualmente podrían manifestar necesidades de apoyo, sino más bien a la que posee un determinado diagnóstico.

Como forma de abordar la diversidad y brechas socioeconómicas del país, en el año 2008 entra en vigencia la Ley 20.248 de subvención escolar preferencial (SEP) (Ministerio de Educción, 2008). Esta ley busca entregar recursos diferenciados al estudiantado, acorde con las condiciones socioeconómicas de las familias, a cambio que las escuelas utilicen estos recursos para asegurar la asistencia a la escuela del llamado alumnado prioritario, y generar mejores resultados en su rendimiento del estudiantado, el cual es medido con la prueba estandarizada SIMCE (Murnane, Page y Vegas, 2009). Las escuelas que se suscriben voluntariamente a esta ley obtienen mayores recursos, los cuales pueden ser utilizados en la contratación de equipos interdisciplinarios para trabajar temáticas de índole psicosocial. A partir de ello, y mediante la incorporación de la temática de convivencia escolar dentro de los planes de mejoramiento educativo (PME), se ha abierto campo a la incorporación de otros grupos profesionales que permitan disminuir las brechas de acceso al aprendizaje (Cádiz y Manríquez, 2015). En este sentido, la SEP sugiere la incorporación de asistencia psicológica y social para el mejoramiento del clima y convivencia escolar, mediante el fortalecimiento de la relación familia-escuela y el vínculo educativo-afectivo con estudiantes (Art n. 8 Ley N. ${ }^{\circ}$ 20.248) (Ministerio de Educación, 2008).

Así también, en el año 2011 entra en vigencia la Ley 20.536 sobre violencia escolar (LSV) (Ministerio de Educación, 2011b), la que regula las situaciones de violencia en las escuelas. Desde esta ley se entiende por convivencia escolar la coexistencia pacífica de miembros de la comunidad educativa, que permita unainteracción positiva, lo que a su vez, favorezca eladecuado cumplimiento de los objetivos educativos en un clima que propicie el desarrollo integral del estudiantado (art. 16a). Posteriormente, en el año 2015, se desarrolla La Política de Convivencia Escolar 2015-2018 (Ministerio de Educación, 2015), que pone en el centro de interés al estudiantado y sus procesos de aprendizaje, al declarar la necesidad de generar "apoyos consistentes que erradiquen modelos [homogenizantes], criminalizadores y judicializantes y se encaminen hacia modelos comunitarios" (MINEDUC, 2015, p. 50). Para su funcionamiento, esta ley establece que en cada escuela debe existir un persona encargada de convivencia escolar, y solo queda a nivel de sugerencia que estos sujetos actores trabajen de manera colaborativa e interdisciplinaria con otros (MINEDUC, 2015), como, por ejemplo, con profesionales que trabajan en el marco de le ley SEP.

Todas estas políticas deben ser plasmadas en los distintos instrumentos de gestión escolar tales como: a) el proyecto educativo institucional (PEI), en el cual se definen los principios orientadores de la formación en escuela; y b) el plan de mejoramiento continuo (PME), en el que se fija la ruta de trabajo que guiará el quehacer educativo (Agencia de calidad de la Educación, 2017). 
doi: http://dx.doi.org/10.15359/ree.23-3.12

URL: http://www.una.ac.cr/educare

CORREO: educare@una.cr

\section{Avances en estudios de políticas educativas: Una mirada de conjunto}

En un reciente artículo, López, González et al. (2018) señalan tres aspectos críticos presentes en la política educativa en Chile: 1) una nueva arquitectura de la política educativa basada en la rendición de cuentas, con altas consecuencias para las escuelas; 2) preeminencia de enfoques punitivos por sobre formativos, 3) predominio de perspectivas integracionistas en desmedro de enfoques inclusivos.

En concreto, respecto a la ley SEP, se advierte que en esta ley subyace una tensión entre una lógica que propicia la autonomía en la gestión escolar, junto con otra de amenaza y sanción, como es el cierre de la escuela (Ahumada, 2010; Assaél et al., 2014). Desde esta perspectiva se hace un fuerte cuestionamiento a la lógica de la rendición de cuentas de altas consecuencias, lo que genera efectos no previstos en sus intenciones originales de efectividad y eficiencia de las escuelas, tales como: favorecer el agobio y la tensión en el personal docente por la responsabilidad de no conseguir buenos resultados, y estigmatizar a las escuelas (Assaél et al., 2012), así como también, focalizar el quehacer diario en conseguir las evidencias como forma de responder a la política (Falabella, 2014).

En cuanto a la ley de convivencia escolar, las investigaciones han dado cuenta de que existe una tensión entre enfoques punitivos, cuyo objeto de intervención es la violencia escolar, y el castigo y la sanción su forma de abordaje; y, por otra parte, un enfoque formativo, que busca resolver los conflictos mediante el diálogo y la reparación (Carrasco, López y Estay, 2012; López, Ramírez, Valdés, Ascorra, y Carrasco-Aguilar, 2018; Magendzo, Toledo y Gutiérrez, 2013). Esta perspectiva punitiva y de amenaza también es relacionada con la superintendencia, organismo que aparece como una figura amenazante, al desempeñar la función de fiscalización de las escuelas, propiciando así un ambiente de política educativa punitiva (Avalos y Assael, 2006).

Por otra parte, si bien Chile avanzó en términos normativos desde un enfoque integracionista hacia uno inclusivo, plasmado en la Nueva Política de Educación Especial creada el año 2005, aún prevalece una lógica individual, más acorde con un modelo bio-médico (Centro de Innovación en Educación, Fundación Chile y Ministerio de Educación, 2013; López et al., 2014), en el cual el foco está puesto en generar estrategias que posibiliten a los individuos adaptarse a las regulaciones escolares, y lograr permanecer en el sistema (López, González et al., 2018). Finalmente, son pocos los estudios en torno a profesionales del ámbito psicosocial que se han comenzado a integrar a las escuelas en el marco de la Ley Sep, pero hasta ahora se ha señalado como importante, que se debe avanzar en la definición de sus funciones y rol dentro de la escuela (Gatica, 2016) con el fin de evitar que reproduzcan enfoques integracionistas (López, González et al., 2018). Las investigaciones que se han realizado sobre el PIE han girado en torno a los efectos y consecuencias de su implementación (Centro de Innovación en Educación, 
Fundación Chile y Ministerio de Educación, 2013; López, Julio, Morales, Rojas y Pérez, 2014); y en relación con el mejoramiento de las propias prácticas pedagógicas del programa de integración escolar (Rodríguez, 2012; Rodríguez y Ossa, 2014; Urbina, Basualto, Durán y Mirtanda, 2017).

De acuerdo con esta revisión, las investigaciones han estado centradas en analizar la implementación y los efectos de cada una de estas leyes y normativas en el ámbito escolar; sin embargo, no hay evidencia suficiente sobre la relación y articulación entre estas distintas políticas en las escuelas. Ello hace importante analizar las traducciones y articulación de estas políticas en equipos de apoyo al estudiantado.

\section{Traducciones de la política y prácticas escolares}

La política pública genera una serie de leyes, normativas e instrumentos pertinentes para su puesta en marcha; sin embargo, esta se pone en juego en su implementación, la cual está atravesada por diferentes actores, en redes locales heterogéneas que, como señala Grau et al. (2010), confluyen en "una red híbrida que se articula en una arena en la que ya existen otras redes" (p. 66), lo que implica un proceso denominado recontextualización, en el cual, "tanto el contexto externo como el interno [en el cual] se sitúan los agentes educativos resultan clave para entender sus respuestas ante cualquier tipo de política" (Verger et al., 2016, p. 7).

La teoría del actor-red (Latour, 1998) entrega una herramienta teórica y de aproximación metodológica que permite estudiar las políticas y prácticas educativas, entendidas como una red fluida de actantes, construida tanto por sujetos como por objetos que pueden ser abordados por las leyes en sus términos declarativos, como los procedimientos e instrumentos que instituyen, y de cómo estos se relacionan y adquieren nuevos sentidos en el ámbito local.

Desde esta mirada, nos centramos en las interacciones entre entidades humanas, en este caso, actores educativos, y no humanos, como las políticas, y sus consecuentes programas, enfatizando que el poder y las negociaciones que entre sí ejercen van configurando una red relativamente estable (Latour, 2005, en Grau, et al., 2010). En este sentido, las prácticas educativas locales no son el resultado de una aplicación unidireccional de los mandatos centrales, sino que estas adquieren distintas formas y prácticas que se negocian a nivel local, en las que la implementación de las políticas se entiende como un proceso de traducción que crea y recrea contantemente la red (Feldman, Khademina, Ingram, y Schneider, 2006).

La bibliografía sobre ANT ha definido conceptos relevantes para el análisis de las políticas educativas. Uno de ellos es el de traducción (Callon, 1986; Latour, 1996), entendida como desplazamiento, deriva, invención, mediación, la creación de un lazo que no existía antes y que hasta cierto punto modifica dos elementos o agentes (Latour, 1996). De esta manera, la 
doi: http://dx.doi.org/10.15359/ree.23-3.12

URL: http://www.una.ac.cr/educare

CORREO: educare@una.cr

teoría de actor-red puede ser considerada, entonces, como una sociología de la traducción, una sociología de todas las negociaciones, gracias a las cuales un actor o fuerza toma o se le confiere autoridad para hablar o actuar en nombre de otro actor o fuerza (Latour, 1996).

Otro concepto relevante para el análisis del presente estudio es el de dispositivos. Los dispositivos se entienden como ensamblajes materiales y discursivos que intervienen en la acción local (Muniesa, Millo y Callon, 2007). Los dispositivos pueden ser, entre otros, ciertas técnicas analíticas, indicadores, protocolos, etc. Muniesa et al. (2007) entienden los dispositivos también como ensamblajes materiales y discursivos, pero que intervienen en la acción local. La noción de dispositivo puede resultar útil para la articulación entre práctica y política, ya que puede guardar relación con todos los documentos claves que operan entre la escuela y el marco normativo; "es decir, como actores propiamente tales que hacen cosas y hacen a otros actuar, hacer cosas. Pero, además, como exponen los autores, permite comprender su agencia, como un emergente de complejos procesos de agenciamiento" (Sisto y Zelaya, 2013, p. 1347).

Para ANT, la relación entre políticas ministeriales y prácticas locales puede entenderse como un movimiento de traducción. Los modelos de traducción suponen que las ideas y formas de conocimiento se alteran a medida que se mueven a través del tiempo y a lo largo del espacio (Latour, 1999, 2005). Siguiendo a Gherardi (2009), una práctica se puede entender como una acción colectiva bien informada que forja las relaciones y conexiones entre todos los recursos disponibles y todas las limitaciones que se presenten, en la que se generan ensambles sociotécnicos que alinean a sujetos, artefactos y otros dispositivos, de modo tal que cada elemento adquiere una posición y un sentido en la interacción.

En función de las perspectivas antes señaladas, las preguntas que guiaron el desarrollo de este estudio fueron dos: ¿Cómo las escuelas traducen las políticas educativas?, especialmente las políticas más visibles y presentes en la cotidianeidad de las escuelas. ¿Cómo se articulan estas políticas educativas dentro de los centros escolares?

\section{Metodología}

En el marco de un diseño cualitativo de investigación, el presente estudio es de tipo etnográfico secuencial (Díaz, 2013; Rockwell, 2009), específicamente, una etnografía de dispositivos (Sisto y Zelaya, 2013). Este formato etnográfico permite acceder a un espacio específico dada la relevancia que este espacio o procedimiento tiene para una institución. En el caso de este estudio, el foco etnográfico estuvo puesto en las prácticas del equipo de apoyo al estudiantado que se conformaba en ambas escuelas y que permitían responder a las políticas ministeriales. 


\section{Participantes}

En este estudio participaron dos escuelas particulares subvencionadas. La escuela 1 pertenece ala Región metropolitana, y la escuela 2 pertenecea la Región de Valparaíso. Si bien ambasescuelasson de distintas ciudades, los dos centros educativos atienden a una población proveniente de similares condiciones socioeconómicas y se encuentran en sectores urbanos periféricos. Lo característicos de ambas escuelas, y por lo cual fueron seleccionadas, es porque decidieron conformar un equipo que autodenominan de "apoyo al estudiante", que permite reunir a docentes y profesionales que operan con alguna de las leyes o normativas consideradas en este estudio.

En el caso de la escuela 1, el equipo se conformó hace dos años con el fin de generar un equipo de apoyo al estudiantado. Se encarga y articula todos los programas ministeriales que aluden a temáticas no académicas. El equipo está integrado por profesionales de la orientación, psicología, la coordinadora del programa de integración escolar (PIE), asistente social (parte de la dupla psicosocial), inspectora general, profesora de educación especial, encargada de convivencia y encargada de pastoral. En el caso de la escuela 2, el equipo se conformó hace dos años y nace por recomendación de la jefatura técnica de contar con un grupo que apoyara (de forma académica o no) al estudiantado, y que además monitoreara los programas ministeriales en los cuales está inserto el alumnado. Este equipo lo integra la jefa de unidad técnico-pedagógica (jefa de UTP), psicóloga, profesor jefe de curso, profesora de educación especial y directora.

\section{Procedimientos y técnicas de producción}

El ingreso a las escuelas fue negociado con la encargada de convivencia, en el caso de la escuela 1, y con la directora, en el caso de la escuela 2. El trabajo se extendió por 4 semanas en cada escuela y se fue realizando de manera progresiva conforme con los resultados que se iban produciendo. Tanto para la inmersión etnográfica en general como para el desarrollo de las diversas técnicas específicas, se solicitaron previamente las autorizaciones al equipo directivo de las escuelas participantes, se incorporaron consentimientos informados a personal adulto.

En el caso de la escuela 1, se realizó una entrevista a la encargada de convivencia y observaciones participantes en las reuniones semanales del equipo de apoyo al estudiantado. En el caso de la escuela 2, se realizaron entrevistas a la directora y a la jefa de UTP y también se realizaron observaciones participantes en las reuniones semanales. Todas las entrevistas fueron semiestructuradas previa organización de un guion temático. El énfasis en la entrevista y en la observación estuvieron centrados en dos aspectos: (1) el reconocimiento de las prácticas de este equipo, con el objetivo de conocer cómo es traducida la política desde el equipo; (2) en la articulación de las políticas, especialmente ley SEP, PIE y política de convivencia escolar. 
doi: http://dx.doi.org/10.15359/ree.23-3.12

URL: http://www.una.ac.cr/educare

CORREO: educare@una.cr

\section{Análisis de datos}

Para organizar el análisis de las entrevistas y de las reuniones y, por lo tanto, para ordenar las categorías seleccionadas, utilizamos el análisis de contenido temático (Vázquez, 1996). Mediante una triangulación de técnicas (Flick, 2014) identificamos aquellas prácticas y discursos que permitían responder al objetivo del estudio y se establecieron las siguientes etapas de trabajo: (1) lecturas de datos, (2) codificación abierta, (3) categorización (4) triangulación, (5) reunión del equipo de investigación y (6) consenso de prácticas relevantes en función de la traducción y articulación de politicas.

\section{Resultados}

Desde los primeros análisis y considerando las entrevistas y observaciones realizadas a los equipos de apoyo al estudiantado, emerge como principal resultado la existencia de un dispositivo que hemos denominado CASO, el que opera como un discurso articulador que se hospeda en todo el sistema de prácticas de los equipos, movilizando sus acciones y procedimientos. El caso, así, como palabra aislada, es una expresión que ocupan ambas escuelas para referirse a estudiantes que se consideran "problemas". Es decir, quienes presentan problemas de aprendizaje, conductuales o emocionales. El estudiantado que presenta alguna dificutad es beneficiario de algunas de las políticas estudiadas (Sep, PIE o de convivencia escolar) $y$, por lo tanto, la escuela cuenta con profesionales, recursos asignados y objetivos para este grupo de estudiantes. Este alumnado es relevante para los centros educativos y por esa razón la expresión caso ocupa gran parte de la retórica escolar y está presente en las conversaciones pedagógicas (y no pedagógicas del equipo).

Por la presencia y preeminencia discursiva de CASO entre miembros del equipo, comenzamos a seguir este dispositivo, para analizar cómo se producen y mantienen sus particulares formas de acción. Por esta razón, el análisis, en una segunda etapa, buscó mapear el CASO como dispositivo para conocer cómo permite mediar la traducción y articulación de las políticas anteriormente señaladas.

Para describir cómo media el dispositivo CASO en la traducción de la política educativa, se levantaron dos categorías de análisis: (1) Detección y apoyo a estudiantes con problemas e (2) Investigación y levantamiento de evidencias. Y respecto a cómo este dispositivo opera en el equipo de apoyo al estudiantado y articula las politicas educativas, se consideró pertinente organizar el análisis también en dos categorías. (3) Anticiparse a las fiscalizaciones y denuncias, y (4) Adopción del lenguaje judicial. En síntesis, los resultados se organizan en 4 categorías que permiten responder a los objetivos del estudio. 


\section{Traduciendo las políticas}

\section{Detección y apoyo a estudiantes con problemas}

EI CASO como dispositivo organiza el quehacer del equipo de gestión, pues permite traducir la política en torno a la detección y apoyo hacia aquel estudiantado que presente algún problema en la escuela y que, por lo tanto, es beneficiario de la normativa vigente. Como se observó, los equipos se reúnen entre dos y tres horas a la semana, con el propósito de revisar las situaciones problemáticas que existen en cada uno de los cursos, cuya conversación está guiada por una persona encargada del equipo, quien comienza preguntando: ¿Qué caso tenemos en ese curso? Desde esta pregunta el resto del equipo comienza a señalar al estudiantado que tiene algún problema, ya sea de comportamiento, rendimiento académico, o de baja asistencia, tal como se señala:

El equipo de apoyo se reúne todas las semanas y conversamos casos de alumnos, en particular que tengan dificultades en el área social, académico o conductual, y se hace un plan de acción para ese alumno, con las necesidades de ese alumno para apoyarlo y que le vaya bien. (Orientadora, escuela 1)

En el mismo contexto, la jefa de UTP comenta que una de las tareas principales de este equipo es poder detectar situaciones problemáticas y poder definir en conjunto las acciones que se deben realizar para su resolución, al respecto especifica:

Le doy un formato al profesor donde me presenta los casos más complejos en términos de rendimiento, disciplina o asistencia, que sean casos para buscar resoluciones. (Jefa de UTP, escuela 2)

En este sentido, las reuniones de los equipos se organizan en torno a la detección de lo que denominan el CASO, que en términos discursivos se constituye como una situación problemática que presente algún estudiante, ya sea un déficit académico o socioemocional, o porque no se adecua o transgrede las normas y a la autoridad, quien, por ende, requiere algún tipo de apoyo. Al respecto, la encargada de convivencia señala que en la escuela asisten niños y niñas que traen problemas desde sus casas, que necesitan de apoyo por parte de este equipo:

Hay niños que tienen problemas con la resolución de conflictos como por costumbre de donde viven, entonces nuestro plan de convivencia parte desde lo más básico que es primero tratar de enseñarles a resolver conflictos. (Encargada de convivencia, escuela 1)

En este sentido, el equipo opera desde el entendido de que en la escuela existen estudiantes que tienen algún tipo de déficit o condición de educación especial, que debe atender y resolver, tal como se señala: 
doi: http://dx.doi.org/10.15359/ree.23-3.12

URL: http://www.una.ac.cr/educare

CORREO: educare@una.cr

En la escuela, tenemos también el niño vulnerable, el prioritario, el niño con necesidades especiales. (Psicóloga, Escuela 2)

Es así como también desde este dispositivo se configura el rol y función de miembros del equipo, pues tal como operan sus reuniones, al identificar al estudiante que presenta alguna situación problemática, posteriormente se define quiénes se harán cargo de cada estudiante y de qué manera, tal como señalan:

Pude coordinar con un profesor, pero desde la voluntad y ahí la P. hace una reunión con los que se designaron tutores y les dice "miren, ustedes son tutores" "a ti te toca este niño y éste", y les cuenta en qué consiste, les dice que lo acompañen, que lo guíen, que le vean las notas, y definan si tiene que reforzar, que igual te puedes apoyar en otras personas, pero es para que el niño se sienta acompañado (Jefa de UTP, escuela 2)

En síntesis, el dispostivo CASO organiza el quehacer de los equipos, en el cual una primera forma de traducir las políticas es trabajar y focalizarse en la detección de "estudiantes-problemas", para generar los apoyos necesarios. Este alumnado, al ser beneficiario de más de una política educativa, permite a la escuela facilitar la gestión, coordinación y también la intervención sobre estudiantes. Por lo tanto, contribuye a las negociaciones que se toman dentro del equipo de apoyo al estudiantado.

\section{Investigación y levantamiento de evidencias}

El CASO como dispositivo también define la forma en la cual miembros del equipo deben realizar sus acompañamientos y apoyos a estudiantes. Luego de la distribución de tareas y asignaciones de estudiantes con algún problema, comienza un proceso que denominan de "investigación", que implica recabar antecedentes de la situación problemática y realizar un resgistro escrito de la información, así como también, de las medidas que se tomen. Esto queda bien señalado cuando la encargada de convivencia da cuenta de los procedimientos que realiza con estudiantes que presentan alguna dificultad:

Cuando un niño amenaza a otro yo abro una investigación, le pongo arriba Caso $x$, en situación esto y esto, los alumnos tantos, el alumno x indica esto y esto, y la medida disciplinar será esta, se asume una mediación si es que es necesario un acto de reparación. (Encargada de convivencia escolar, escuela 1)

Como parte del proceso de investigación, además se activan una serie de protocolos e instrumentos de planificación y seguimiento, tales como el "informe escolar","hoja de derivación", "ficha pro-retención", o"ficha de mediación", utilizados por miembros del equipo como medios de verificación de sus acciones. Un ejemplo concreto es la "ficha de derivación" que se utiliza en la escuela 1 (Figura 1), la cual sirve para consignar aspectos relevantes del estudiantado. 
doi: http://dx.doi.org/10.15359/ree.23-3.12

URL: http://www.una.ac.cr/educare

CORREO: educare@una.cr

\section{FICHA DERIVACIÓN (ESTUDIANTE)}

EQUIPOS DE APOYO

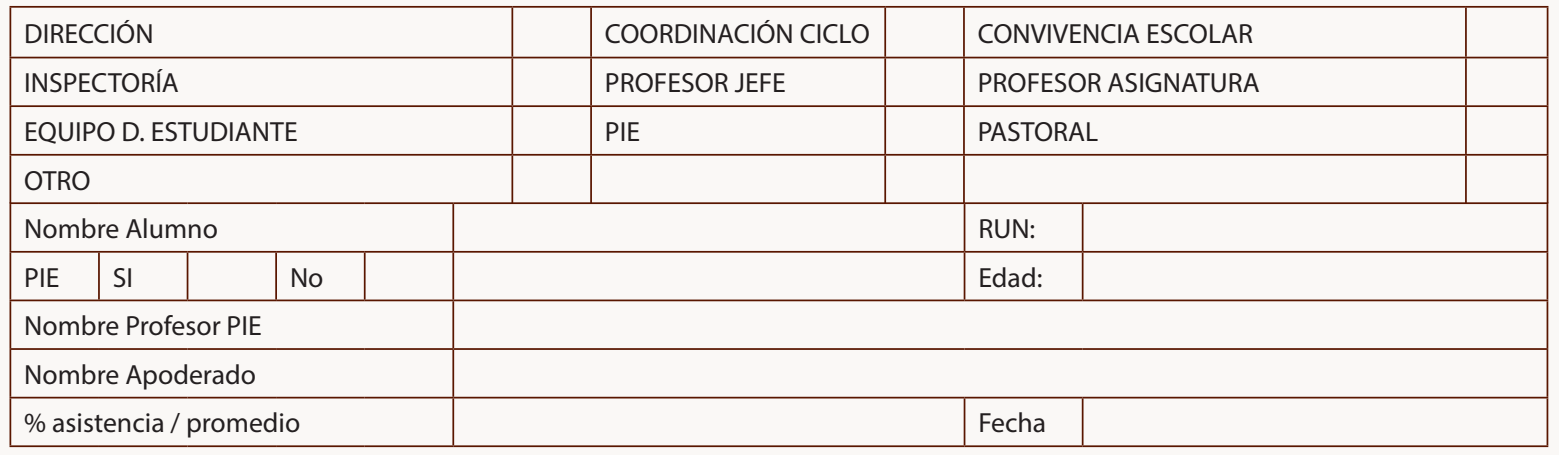

MOTIVO DE LA DERIVACIÓN: (Características y situación del alumno, especificar urgencia, otras)

ACCIÓN REALIZADA POR PERSONAS QUE DERIVA: (entrevista del alumno, contacto con el apoderado, etc.)

\begin{tabular}{|l|l|l|}
\hline Nombre: & Nombre: & Recepcionar: \\
RUN: & RUN: & RUN: \\
Firma: & Firma: & Firma: \\
\hline
\end{tabular}

(USO INTERNO Equipos de Apoyo)

Profesional Responsable de EA

Fecha de recepción derivación

Figura 1: Ficha de derivación.

Nota: Instrumento interno de la escuela 1. 
doi: http://dx.doi.org/10.15359/ree.23-3.12

URL: http://www.una.ac.cr/educare

CORREO: educare@una.cr

Al revisar los ítems que contempla este instrumento, se oberva que este se utiliza para consignar los siguientes aspectos: 1 ) identificar quiénes están a cargo de cada estudiante con problemas; 2 ) identificar la pertenencia o no de cada estudiante al programa de integración ( PIE); 3) señalar el porcentaje de asistencia, entendido como información relevante para los programas de retención de estudiantes en la escuela; 3) señalar la situación problemática de cada estudiante; 4) señalar las acciones que ha realizado la persona que deriva; y 5) dejar registro, a través de la firma, de todas las personas de la escuela que han sido parte de este proceso.

De esta manera, el dispositivo caso actualiza una serie de procedimientos e instrumentos de gestión, que para los sujetos integrantes del equipo resultan relevantes como medios de verificación de las acciones que realizan. En este mismo sentido, uno de los aspectos que más señalan los equipos es que, una vez que se ha detectado un problema y comienza el proceso de investigación, deben dejar registrado todo lo que hacen, lo cual denominan "evidencias". En este sentido, el dispositivo CASO moviliza a cada miembro del equipo hacia el levantamiento de evidencias de su quehacer, tal como se señala:

Bueno y esa tensión se traspasa a nuestra mesa finalmente porque cada uno tiene que velar por lo que tenemos que responder finalmente, entonces eso hace que esa tensión también se traspase, porque uno entiende el área social por ejemplo, pero yo tengo que cumplir con mi evidencia y la inspectora tiene que cumplir con sus medidas disciplinares, y el orientador debe tener sus evidencias. (Psicóloga, Escuela 1)

Resumiendo, una segunda forma de traducir las políticas es trabajar y focalizar las prácticas de estos equipos en el levantamiento de evidencias. Ambas escuelas funcionan con documentos de trabajo que no solo orientan la toma de decisiones sobre el alumnado, sino que permite ordenar la información en función de las políticas educativas. Esta lógica de trabajo facilita la traducción, pues todas las políticas que antes se mencionan se sostienen en documentos de respaldo a la hora de la fiscalización por parte de organismos examinadores.

\section{Articulando las políticas}

\section{Anticiparse a las fiscalizaciones y denuncias}

Una primera forma de articular las distintas políticas es centrar el quehacer del equipo en generar las evidencias que le permitan anticiparse a las fiscalizaciones o posibles denuencias. Como hemos señalado, los equipos de apoyo al estudiantado se organizan en torno a la detección de estudiantes con problemas, y para eso se echa a andar una serie de procedimientos, los cuales explican, son relevantes en caso de fiscalizaciones o denuncias que puedan hacer las personas apoderadas en la Superintendencia. Al respecto, la encargada de convivencia señala: 
Si unos muchachos tuvieron una pelea queda escrito y firmado y solucionamos el conflicto, les hago firmar como un acta de mediación en la que hacen incluso acuerdo, por ejemplo me comprometo a si me pegas una patada sin querer en el futbol voy a tratar de contar hasta diez para no pegarte un combo, casi todo -porque a veces se escapan-todo queda por escrito y firmado, de todo tratamos de hacer evidencia. Uno porque para uno es importante para después corregirse, revisar lo que está haciendo, potenciar lo bueno, y lo otro porque la Superintendencia en caso de una denuncia o reclamo te pide todo, entonces tiene que haber evidencia de todo. (Encargada de convivencia escolar, Escuela 1)

Así también, el seguir ciertos procedimientos y generar evidencias, es entendido como parte central de su quehacer, puesto que está en sintonía con la forma de operar del Ministerio de Educación o Superintendencia, desde donde viene protocolizado y se debe responder acorde con ello, como se comenta:

Hay que entender que todas las políticas públicas bajan para nosotros a través del RICE (Reglamento interno de convivencia escolar) del manual de convivencia, que todo lo que está estipulado ahí es lo que nosotros podemos dejar en evidencia ante el Ministerio o ante la Superintendencia, y dentro del RICE están los protocolos, los deberes y derechos, el manual de convivencia, los planes de gestión. (Inspectora, escuela 1)

En síntesis, el dispositivo CASO articula las politicas en torno al levantamiento de evidencias, que les permiten a los equipos estar preparados para las fiscalizaciones que realiza la Superintendencia, o para poder enfrentar posibles denuncias. Para cualquiera de las dos situaciones, resulta central contar con los registros de las acciones y procedimientos realizados, por lo que la anticipación a la fiscalización o denuncia se levantan como prácticas claves para articular las politicas que están destinadas a la escuela.

\section{Adopción del lenguaje judicial}

Una segunda forma de articular las políticas es jugando con las reglas discursivas de las mismas políticas. Es decir, usando y haciendo suyo el mismo lenguaje legislativo. Para los sujetos miembros de los equipos, esto permite apropiarse del espíritu de las actuales políticas que rigen en educación. Para esto el dispositivo CASO resulta útil, pues amarra diversas políticas, que si bien tienen fines distintos, se rigen con los mismos procedimientos legales para su rendición o monitoreo. Al respecto, la encargada de convivencia señala:

Lo que pasa es que se en algún minuto todo el lenguaje educacional cuando se tienen que aplicar normas de convivencia escolar en esto de la ley de violencia, se empezó a hablar en muchos términos legales... y la de violencia empezó a generar esto o sea de hablar de caso, de sanción de medidas disciplinares. (Encargada de convivencia, escuela 1). 
doi: http://dx.doi.org/10.15359/ree.23-3.12

URL: http://www.una.ac.cr/educare

CORREO: educare@una.cr

Por tanto, el Caso como dispositivo ha permitido enfrentar una nueva configuración discursiva que se ha instalado en la escuela, para lo cual se activan distintas acciones y mecanismos que lo sustenten y demuestren las acciones realizadas por la escuela, pues como señala el orientador, el mundo de la educación ya ha adquirido una lógica de trabajo basada en evidencias, en sus palabras:

Es que yo creo que ya es una lógica de trabajo, como hiciste algo demuéstrelo, la foto como que ya se dejó de lado pero tiene que estar, pero síque firmen las personas que asistieron, si es que hay una tabla de trabajo. (Orientador, escuela 1)

En este sentido, desde la perspectiva del equipo, se ha transmutado, desde el pedagógico, hacia un lenguaje judicial, como señala la encargada de convivencia:

Además que la Superintendencia o el Ministerio de Educación siempre cuando te entregan un oficio, resolución exenta, o resolución, respuesta a su resolución, entonces todo el lenguaje se ha ido transformando. (Encargada de convivencia, escuela 1).

En síntesis, para los equipos de apoyo, el CASO como dispositivo ha cristalizado el lenguaje judicial que opera fuertemente en el ámbito educativo, y esto propicia que todo el equipo de apoyo al estudiantado se articule en torno a la detección, investigación y levantamiento de evidencias.

\section{Conclusiones}

Este estudio tuvo por objetivo dar cuenta de cómo las escuelas traducen y articulan la diversidad de políticas que operan en el sistema, tales como la Ley SNAC, Ley SEP, Ley de convivencia escolar y PIE. Para ello, la inmersión etnográfica se focalizó en dos escuelas que tenían en su interior un equipo de apoyo al estudiantado. Este equipo organizaba la presencia de diversas políticas que tenían, muchas veces, al mismo estudiantado como beneficiario.

Si bien la sola presencia del equipo es una forma de entender la traducción de las políticas, lo cierto es que producto de los análisis, de las visitas y de las entrevistas, emerge un dispositivo que denominamos $C A S O$, desde el cual se organiza el quehacer de los equipos, principalmente en torno a la resolución de conductas problemáticas de estudiantes, que les permitan mantenerles en la escuela, como forma de responder a la lógica de rendición de cuentas que opera en la gestión educativa.

Le atribuimos el componente de dispositivo, pues ensambla discursos, acciones y recursos a nivel escolar, interpelando a la escuela a través de su relación con las políticas locales. El CASO 
como dispositivo permite el desplazamiento de las políticas ministeriales a la realidad escolar, desde el cual se generan ciertas negociaciones, lazos, la mediación y, por lo tanto, la agencia del diverso grupo de profesionales que componen el equipo de apoyo al estudiantado. La sola palabra "caso" da por hecho una serie de significados intrínsecos que ordena la labor pedagógica, pero también la labor política.

Este dispositivo, en su función mediadora, presencial y preeminente, no solo contiene la facilidad de permitirles a las escuelas traducir las políticas escolares de apoyo, sino además se convierte en un escenario para articular las diversas políticas. De ese modo, el objetivo de este estudio se logra (y se entiende) desde el funcionamiento del CASO como dispositivo al interior de los dos centros escolares, de este se analiza cómo media en la traducción de las politicas educativas, y en la articluación de estas políticas en el equipo de apoyo al estudiantado.

Respecto a la traducción de las políticas educativas, se levantaron dos categorías de análisis: (1) Detección y apoyo a estudiantes con problemas; la cual hace referencia a que el dispositivo CASO funciona como organizador del quehacer de los equipos, traduciendo las las políticas en torno a la focalización y detección de "estudiantes-problemas", y (2) Investigación y levantamiento de evidencias; que da cuenta de cómo, a partir de este dispositivo, el quehacer de los equipos está centrado en el levantamiento de evidencias.

En relación con la articulación de las políticas educativas, se levantaron también dos categorías: (1) Anticiparse a las fiscalizaciones y denuncias; refiere a que desde el dispositivo CASO, la articulación de la política está puesta en contar con evidencias que permitan anteponerse a las fiscalizaciones o posibles denuncias, y dialogar con los organismos educativos centrales, y (2) Adopción del lenguaje judicial; referida a que la articulación de la política se realiza usando y haciendo suyo el mismo lenguaje legislativo, que explica el uso de la palabra caso, investigación y evidencias. Para las escuelas resulta útil apropiarse de esta nueva nomenclatura, pues si bien las diversas políticas tienen fines distintos, se rigen con los mismos procedimientos legales para su rendición o monitoreo.

Considerando estos resultados, se advierte que estos equipos de apoyo a estudiantes traducen lo político y lo articulan acorde con una visión managerial de los servicios públicos, en la que importan los indicadores y resultados de rendimiento que, en definitiva, permiten responder a otros dispositivos que operan en la política educativa en Chile, tales como el SIMCE o rendición de cuentas (Assaél et al., 2014; López, González et al., 2018).

Lo anterior se explica por varias razones: 1) es importante como escuela resguardarse de la fiscalización de la Superintendencia, así como también, de las posibles denuncias que puedan hacer los sujetos apoderados; 2) las escuelas requieren tener buenos indicadores para acceder a los recursos y subvenciones del Estado; y, a su vez 3 ) los indicadores son importantes para sobrevivir al fantasma del posible cierre de la escuela (Ahumada, 2010; Assaél et al., 2014). 
doi: http://dx.doi.org/10.15359/ree.23-3.12

URL: http://www.una.ac.cr/educare

CORREO: educare@una.cr

Por tanto, estos equipos optan por responder a lo urgente y centrarse en la resolución de situaciones problemáticas. Así, centran su quehacer en la detección de los casos problemas, que pueden ser de distinta índole, y que responden a distintas normativas, tales como: 1) resolver problemas de comportamiento, que le importan a la política de convivencia; 2 ) resolver problemas de asistencia, que le importan a la Ley Sep; 3 ) resolver problemas de aprendizaje, que le importan al PIE. Por tanto, el quehacer de estos equipos está centrado en la detección, investigación y resolución de problemas individuales, en desmedro de estrategias más preventivas o promocionales con toda la comunidad educativa.

Estos resultados concuerdan con investigaciones que señalan la preeminencia de perspectivas integracionistas en la escuela, cuyo foco está puesto en lograr la adaptación individual; junto con el predominio de un enfoque biomédico, tras el cual las escuelas se centran en los déficits, los diagnósticos y los tratamientos individuales (González, Luzón y Torres, 2012; UNESCO, 2008). Por otra parte, acorde con lo que señalan López et al. (2018), en la política de convivencia se advierte un enfoque punitivo, cuyo énfasis está puesto en la detección, investigación y definición de sanciones para estudiantes que presentan problemas de comportamiento.

Para finalizar, es conveniente precisar que esta investigación se realizó en dos escuelas, con sus características distintivas en la configuración de su equipo, por lo cual adquieren valor en sí mismas, pero no pretende ser generalizable a otras realidades.

A su vez, como hemos planteado, las políticas educativas en los espacios locales se articulan y adquieren particularidades relevantes de analizar, donde la propuesta teórica y metodológica de Sisto y Zelaya (2013) resulta una herramienta que permite comprender las traducciones y sus consecuentes prácticas locales, desde las que se coordinan acciones pertinentes; pero creemos que este tipo de trabajo se enriquecería en la medida en que se consideren las prácticas de otros equipos de trabajo en el ámbito escolar.

En cuanto a las proyecciones, conviene seguir profundizando en las traducciones que hacen las escuelas no solo de las políticas nacionales, sino de las regionales, pues en el sistema escolar chileno, al ser municipalizado, rigen normativas locales y situadas que tienen efectos administrativos y pedagógicos sobre las escuelas.

\section{Agradecimientos}

Centro de investigación para la educación inclusiva, PIA Conicyt CIE 160009, Pontificia Universidad Católica de Valparaíso, y Beca N. ${ }^{\circ} 21190891$ y N. ${ }^{\circ} 21170256$ Doctorado nacional, Conicyt. 


\section{Referencias}

Agencia de Calidad de la Educación. (2017). Los indicadores de desarrollo personal y social en los establecimientos educacionales chilenos: Una primera mirada. Santiago-Chile. Recuperado de $\quad$ http://archivos.agenciaeducacion.cl/estudios/Estudio Indicadores desarrollo personal social en establecimientos chilenos.pdf

Ahumada, L. (2010). Liderazgo distribuido y aprendizaje organizacional: Tensiones y contradicciones de la Ley de Subvención Escolar Preferencial en un contexto rural. Psicoperspectivas. Individuo y Sociedad, 9(1), 111-123. doi: https://doi.org/10.5027/ psicoperspectivas-Vol9-Issue1-fulltext-99

Assaél, J., Acuña, F., Contreras, P. y Corbalán, F. (2014). Transformaciones en la cultura escolar en el marco de la implementación de políticas de accountability en Chile. Un estudio etnográfico en dos escuelas clasificadas en recuperación. Estudios Pedagógicos 40(2), 7-26. doi: http://dx.doi.org/10.4067/S0718-07052014000300001

Assaél, J., Contreras, P., Corbalán, F., Palma, E., Campos, J., Sisto, V. y Redondo, J. (2012). Ley SEP en escuelas municipales emergentes: ¿Cambios en la identidad docente? Paulo Freire. Revista de Pedagogía Crítica, 11(11), 219-228. doi: https://doi.org/10.25074/07195532.11.453

Avalos, B. y Assael, J. (2006). Moving from resistance to agreement: The case of the Chilean teacher performance evaluation. International Journal of Educational Research, 45(4-5), 254-266. doi: https://doi.org/10.1016/j.ijer.2007.02.004

Cádiz, J. y Manríquez, L. (2015). Configuración del trabajo interprofesional en psicólogos y trabajadores sociales en establecimientos municipales enmarcados en Ley SEP (Tesis de licenciatura). Pontifica Universidad Católica de Valparaíso, Chile.

Callon, M. (1986). Some elements of a sociology of translation: Domestication of the scallops and the fishermen of St Brieuc Bay. En J. Law (Ed.), Power, action and belief. A new sociology of knowledge? (pp. 196-223). London: Routledge. \& Kegan Paul.

Carrasco, C., López, V. y Estay, C. (2012). Análisis crítico de la Ley de Violencia Escolar de Chile. Psicoperspectivas. Individuo y Sociedad, 11(2), 31-55. Recuperado de https://doi. org/10.5027/psicoperspectivas-vol11-issue2-fulltext-228

Centro de Innovación en Educación, Fundación Chile y Ministerio de Educación. (2013). Análisis de la complementación de los programas de integración escolar (PIE) en establecimientos que han incorporado estudiantes con necesidades educativas especiales transitorias (NEET). Santiago: Autores. Recuperado de http://www.mineduc.cl/usuarios/edu.especial/ doc/201402101719500.InformeEstudiolmplementacionPIE2013.pdf 
doi: http://dx.doi.org/10.15359/ree.23-3.12

URL: http://www.una.ac.cr/educare

CORREO: educare@una.cr

Cornejo, R. (2006). El experimento educativo chileno 20 años después: Una mirada crítica a los logros y falencias del sistema escolar. Revista Electrónica Iberoamericana sobre Calidad, Eficacia y Cambio en Educación, 4(1), 118-129. Recuperado de http://www.rinace.net/arts/ vol4num1/art8.pdf

Díaz, Á. (2013). Etnografía de la escuela más allá de la etnografía y de la escuela: Tensiones disciplinares y aplicabilidad de los saberes etnográficos. Educación y Futuro, 29, 13-39. Recuperado de https://dialnet.unirioja.es/servlet/articulo?codigo=4456805

Falabella, A. (2014) The performing school: The effects of market $\&$ accountability policies. Education Policy Analysis Archives, 22(70), 1-29. doi: http://dx.doi.org/10.14507/epaa. v22n70.2014

Feldman, M. S., Khademina, A. M., Ingram, H. y Schneider, A. S. (2006). Ways of knowing and inclusive management practices. Public Administration Review, 66(especial 1), 89-99. doi: https://doi.org/10.1111/j.1540-6210.2006.00669.x

Flick, U. (2014). An introduction to qualitative reserch. Berlín: Sage.

Gatica, F. (2016). Las intervenciones psicosociales en establecimientos educacionales municipales vulnerables bajo el marco de la Ley SEP. Diseño, implementación y logros desde la perspectiva de actores claves. Revista Estudios de Políticas Públicas, 2(1), 105-119. doi: http://dx.doi.org/10.5354/0719-6296.2016.41830

Gherardi, S. (2009). Introduction: The critical power of the 'practice lens'. Management Learning, 40(2), 115-128. doi: https://doi.org/10.1177/1350507608101225

González, J. C., Luzón, A. y Torres, M. (2012). Social exclusion in educational discourse: An analysis based on a program of investigation. Education Policy Analysis Archives, 20(24), 1-24. doi: https://doi.org/10.14507/epaa.v20n24.2012

Grau, M., L. Íñiguez-Rueda, L. y Subirats, J. (2010). La perspectiva sociotécnica en el análisis de políticas públicas. Psicología Política, 41, 61-80. Recuperdo de https://www.uv.es/garzon/ psicologia\%20politica/N41-4.pdf

Latour, B. (1996). On actor-network theory: A few clarifications. Soziale Welt, 47(4), 369381. Recuperado de http://transnationalhistory.net/interconnected/wp-content/ uploads/2015/05/Latour-Actor-Network-Clarifications.pdf

Latour, B. (1998). De la mediación técnica: Filosofía, sociología, genealogía. En M. Domènech y F. J. Tirado (Comps.), Sociología simétrica. Ensayos sobre ciencia, tecnología y sociedad (pp. 130-302). Barcelona: Gedisa. 
Latour, B. (1999) On recalling ANT. In J. Law y J. Hassard (Eds.), Actor network theory and after (pp. 15-25). Oxford: Blackwell Publishers.

Latour, B. (2005). Reassembling the social. An introduction to actor-network-theory. New York: Oxford University Press. Recuperado de http://droit-public.ulb.ac.be/wp-content/ uploads/2013/04/Latour Reassembling.pdf

López, V., González, P., Manghi, D., Ascorra, P., Oyanedel, J. C., Redón, S., ... y Salgado, M. (2018). Políticas de inclusión educativa en Chile: Tres nudos críticos. Archivos Analíticos de Políticas Educativas, 26(157), 1-24. doi: http://dx.doi.org/10.14507/epaa.26.3088

López, V., Julio, C., Morales, M., Rojas, C. y Pérez, M. V. (2014). Barreras culturales para la inclusión: Políticas y prácticas de integración en Chile. Revista de Educación, 363, 256-281. doi: 10.4438/1988-592X-RE-2012-363-180

López, V., Ramírez, L., Valdés, R., Ascorra, P. y Carrasco-Aguilar, C. (2018). Tensiones y nudos críticos en la implementación de la(s) política(s) de convivencia escolar en chile. Calidad en la educación, 48, 96-129. doi: http://dx.doi.org/10.31619/caledu.n48.480

Magendzo, A., Toledo, M. I. y Gutiérrez, V. (2013). Descripción y análisis de la Ley sobre Violencia Escolar (N.․ 20.536): Dos paradigmas antagónicos. Estudios Pedagógicos, 39(1), 377-391. doi: https://doi.org/10.4067/S0718-07052013000100022

Ministerio de Educación. (2005). Política nacional de educación especial. Santiago, Chile: Autor. Recuperado de https://www.diversidadinclusiva.com/wp-content/uploads/2015/10/ Pol\%C3\%ADtica-Nacional-de-Educaci\%C3\%B3n-Especial-2005.pdf

Ministerio de Educación. (31 de julio, 2007). Ley 20.201. Modifica el DFL N.o 2, de 1998, de educación, sobre subvenciones a establecimientos educacionales y otros cuerpos legales. Promulgación 06 de junio de 2007. Recuperado de https://www.leychile.cl/ Navegar?idNorma $=263059$

Ministerio de Educación. (01 de febrero, 2008). Ley 20.2248. Establece ley de subvención escolar preferencial, promulgada el 25 de enero de 2008. Recuperado de http://www.leychile. $\mathrm{cl} / \mathrm{N} ? \mathrm{i}=269001 \& \mathrm{f}=2012-10-26 \& \mathrm{p}=$

Ministerio de Educación. (27 de agosto, 2011 a). Ley 20.529. Sistema nacional de aseguramiento de la calidad de la educación parvularia, básica y media y su fiscalización. Promulgada 11 de agosto de 2011. Recuperado de https://www.leychile.cl/Navegar?idNorma=1028635

Ministerio de Educación. (17 de setiembre, 2011b). Ley 20.536. Sobre violencia escolar, promulgada 08 de septiembre de 2011. Recuperado de https://www.google.com/search? $q=i C u a ́ l+e s+l a+L e y+S E P \% 3 F \& s a=X \& v e d=2 a h U K E w j H 6 Z S z 6 Y$ hAhXhw1 
doi: http://dx.doi.org/10.15359/ree.23-3.12

URL: http://www.una.ac.cr/educare

CORREO: educare@una.cr

Ministerio de Educación. (2015). Políticanacional de convivenciaescolar 2015-2018.Santiago, Chile: Autor. Recuperado de https://www.mineduc.cl/wp-content/uploads/sites/19/2015/12/ politica-noviembre-definitiva.pdf

Ministerio de Educación. (2016a). La reforma educacional está en marcha. Tu sueño, nuestro propósito. Cuenta Pública 2015. Santiago, Chile: Autor. Recuperado el 15 de mayo de 2017 desde http://sitios.mineduc.cl/Cuenta\%20P/files/assets/common/downloads/ Cuenta\%20P.pdf

Ministerio de Educación. (2016b). Plan de aseguramiento de la calidad escolar 2016-2019. Sistema de aseguramiento de la calidad de la educación. Santiago, Chile: Autor. Recuperado de http://www.siteal.iipe.unesco.org/sites/default/files/chi - plan de aseguramiento de calidad escolar.pdf

Muniesa, F., Millo, Y. y Callon, M. (2007). An introduction to market devices. The Sociological Review, 55(2), 1-12. doi: https://doi.org/10.1111/j.1467-954X.2007.00727.x

Murnane, R. J., Page, L. y Vegas, E. (2009). Distribución de los rendimientos estudiantiles en Chile. Análisis de línea base para la evaluación de la subvención escolar preferencial (SEP). Recuperado de https://docplayer.es/54002251-Distribucion-de-los-rendimientosestudiantiles-en-chile-analisis-de-linea-base-para-la-evaluacion-de-la-subvencionescolar-preferencial-sep.html

Rockwell, E. (2009). La experiencia etnográfica. Historia y cultura en los procesos educativos. Buenos Aires: Paidos.

Rodríguez, F. F. (2012). La percepción del trabajo colaborativo en la gestión curricular de profesores de educación regular y educación especial en programas de integración escolar de la comuna de Tomé (Tesis de maestría). Universidd del Bío-Bío, Chillán, Chile. Recuperado de http:// repobib.ubiobio.cl/jspui/bitstream/123456789/2135/1/Rodriguez Rojas Felipe.pdf

Rodríguez, F. F y Ossa, C. J. (2014). Valoración del trabajo colaborativo entre profesores de escuelas básicas de Tomé, Chile. Estudios Pedagógicos, 40(2), 303-319. doi: https://doi. org/10.4067/S0718-07052014000300018

Sisto, V. y Zelaya, V. (2013). La etnografía de dispositivos como herramienta de análisis y el estudio del managerialismo como práctica local. Universitas Psychologica, 12(4), 1345-1354. doi: https://doi.org/10.11144/Javeriana.UPSY12-4.edha

UNESCO. (2008). La educación inclusiva: El camino hacia el futuro. Una breve mirada a los temas de educación inclusiva. Aportes a las discusiones de los talleres. París: Autor. Recuperado de https://coleccion.siaeducacion.org/sites/default/files/files/9 unesco.pdf 
Urbina, C., Basualto, P., Durán, C. y Miranda, P. (2017). Prácticas de co-docencia: El caso de una dupla en el marco del Programa de Integración Escolar en Chile. Estudios pedagógicos, 43(2), 355-374. doi: https://dx.doi.org/10.4067/S0718-07052017000200019

Vázquez, F. (1996). El análisis de contenido temático. Objetivos y medios en la investigación psicosocial (Documento de trabajo). Barcelona: Universitat Autònoma de Barcelona.

Verger, A., Bonal, X. y Zancajo, A. (2016). Recontextualización de políticas y (cuasi)mercados educativos. Un análisis de las dinámicas de demanda y oferta escolar en Chile. Education Policy Analysis Archives/Archivos Analíticos de Políticas Educativas, 24(27), 1-27. doi: https:// doi.org/10.14507/epaa.24.2098 